\title{
Off-the-Shelf Cell Therapy with Induced Pluripotent Stem Cell-derived Natural Killer Cells
}

Michelle L. Saetersmoen, ${ }^{1}$ Quirin Hammer ${ }^{2}$, Bahram Valamehr ${ }^{3}$, Dan S. Kaufman ${ }^{4}$, KarlJohan Malmberg ${ }^{1,2,5}$

${ }^{1}$ The KG Jebsen Center, University of Oslo, Norway; ${ }^{2}$ Department of Medicine, Huddinge, Karolinska Institute, Sweden; ${ }^{3}$ Fate Therapeutics Inc. San Diego, USA. ${ }^{4}$ University of California San Diego, Department of Medicine; ${ }^{5}$ Institute for Cancer research, Oslo University Hospital, Norway. 


\begin{abstract}
Cell therapy is emerging as a very promising therapeutic modality against cancer, spearheaded by the clinical success of chimeric antigen receptor (CAR) modified $\mathrm{T}$ cells for $\mathrm{B}$ cell malignancies. Currently FDA approved CAR-T cell products are based on engineering of autologous $\mathrm{T}$ cells harvested from the patient, typically using a central manufacturing facility for gene editing before the product can be delivered to the clinic and infused to the patients. For a broader implementation of advanced cell therapy and to reduce costs, it would be advantageous to use allogeneic "universal" cell therapy products that can be stored in cell banks and provided upon request, in a manner analogous to biopharmaceutical drug products. In this review we outline a roadmap for development of off-the-shelf cell therapy based on natural killer (NK) cells derived from induced pluripotent stem cells (iPSCs). We discuss strategies to engineer iPSC-derived NK cells (iPSC-NK) for enhanced functional potential, persistence and homing.
\end{abstract}




\section{Natural killer cells - an ideal template for off-the-shelf cell therapy}

Immunotherapy has become a cornerstone in cancer therapy that includes a broad array of strategies aiming to unleash, direct and boost the patents' own immune system through adoptive transfer of expanded naturally circulating or genetically engineered cytotoxic lymphocytes. Despite the recent clinical breakthroughs, the field is still in its infancy and the potential for identifying new and more effective strategies is huge. The first FDA-approved gene-edited T cell products for lymphoma and leukemia came out on the market only in 2017 and the needs for new therapies targeting resistant cases and other diseases, in particular solid tumors, remains high. A key challenge for a wider implementation of cell therapy is to come around the laborious procedures of identifying HLA-matched healthy related or unrelated donors and harvesting their cells for engineering and infusion to one patient. Although such tailored one-donor onepatient strategies are feasible and have proven effective, they have a significant lag-time to patient delivery, are very expensive and may be less well suited for large-scale implementation of living immunotherapy drugs. Other bottlenecks include difficulties in obtaining large quantities of T cells or NK cells from peripheral blood, inability to uniformly engineer effector cells and poor function of the cells due to exhaustion.[1, 2] A very attractive solution to these problems would be to expand and genetically engineer cytotoxic lymphocytes ( $\mathrm{T}$ cells, $\gamma \delta \mathrm{T}$ cells or NK cells) that can be stored in cell banks and administered across HLA barriers as an off-the-shelf therapy. Off-the-shelf strategies based on $\mathrm{T}$ cells require genetic manipulation and knock down of the endogenous T cell receptor to avoid graft-versus-host disease (GVHD). Such strategies are available and discussed in several comprehensive reviews. [3-7] An alternative cell type is the natural killer (NK) cell. NK cells do not express rearranged receptors and can be easily transferred across HLA barriers without causing GVHD. In fact, under specific circumstances, transfer across HLA barriers trigger NK cell alloreactivity and has been shown to induce remission in acute leukemia and myelodysplastic syndrome.[8-10]

NK cells have an important role in regulating the defenses against virally infected and tumor cells. $[11,12]$ Natural killer (NK) cells were first described in the 1970s by Kiessling et al. for their ability to lyse target cells without prior sensitization. [13-16] Based on patterns of cytotoxicity against panels of tumors cells with different levels of MHC and inspired by the hunt for foreign submarines in the Swedish archipelago,[17] Klas Kärre identified a key principle behind the functional regulation of NK cells, eg. the recognition of targets that lacked expression of MHC class I on their cell surface. [18-20] NK cells therefore provide an alternative immune defense mechanism compared to $\mathrm{T}$ cells and form a second line of defense 
in case tumors cells escape T cell recognition by down-modulating MHC. [20, 21] While this may possibly occur spontaneously during immune surveillance, it is certainly of relevance in the era of immune selection and loss of HLA class I imposed by T-cell based immunotherapies. [22]

Although NK cells share many characteristics with other lymphocytes in the adaptive immune system, NK cells are classified as part of the innate immune system due to their lack of receptors for antigen specificity as a result of gene rearrangement which is seen in $\mathrm{B}$ and $\mathrm{T}$ cells.[23-25] Instead, the activation of NK cells is determined by the integration of signals from an array of germline encoded activating and inhibitory receptors.[26] Many of these receptors, including the MHC binding killer cell immunoglobulin-like receptors (KIR), are acquired during NK cell differentiation and contribute to the functional calibration of the cell during interaction with host cells in a process termed NK cell education.[27] Unlike T cells, NK cells circulate in a primed state and are full of effector molecules, including perforin and granzyme B.[27] NK cells are therefore ideal cellular templates for gene editing approaches aiming at directing their potent cytotoxic potential against a desired target. To harness the full potential of NK cells one needs to consider the cellular mechanisms involved in acquisition of effector function during terminal differentiation and the subsequent fine tuning through education. This is particularly relevant when using iPSCs or cord blood derived CD34 ${ }^{+}$hematopoietic stem cells as a source for NK cells since in vitro differentiation may not always lead to fully mature NK cells. This review discusses several issues that needs to be considered in order to develop efficient platforms for iPSC-derived off-the-shelf NK cell therapy, including specificity, in vivo persistence, homing, resistance to immune suppression and modulation of effector function (Figure 1).

\section{iPSC-derived NK cells}

In 2006 Takahashi and Yamanaka first produced induced pluripotent stem cells by simultaneously introducing four genes to mouse fibroblasts. These reprogramming factors effectively convert mouse fibroblasts to pluripotent stem cells with properties similar to embryonic stem cells (ESCs). [28] A year later, a similar reprogramming method was used to generate iPSCs from human fibroblasts. [28, 29]. While ESCs and iPSCs have similar morphology, growth characteristics and developmental potential, global gene expression analysis of ESCs and iPSCs have shown some persistent donor gene expression in iPSCs, 
indicative of a potential difference between iPSCs and ESC.[30, 31] Likewise, microarrays of human iPSC and ESC lines revealed differentially expressed genes.[32] In addition, several studies showed differences in DNA methylation between ESCs and iPSCs lines as well as an epigenetic imprint of donor cells in human iPSCs. [33-37] However, other studies have attributed the differences in gene expressions to different culture conditions in the laboratory. $[38,39]$

Human embryonic stem cells (hESCs) were initially used to produce human NK cells able to kill diverse tumor cells in vitro.[40] These hESC-derived NK cells were shown to be better than NK cells derived from umbilical cord blood (UCB-NK cells) in killing K562 cells in a murine xenograft model.[41] Therefore, while hESCs also serve as an effective source of off-the-shelf NK cells for allogeneic immunotherapy, iPSCs are easier to obtain and provide potentially greater donor diversity which may be of particular interest for examining influence of KIR haplotypes on adoptive NK cell immunotherapy.

iPSCs can now be routinely generated from a variety of easily obtainable sources such as skin and peripheral blood (PB) and once reprogrammed iPSCs are able to undergo essentially unlimited expansion in vitro without losing pluripotency.[42] Therefore, iPSCs can serve as a resource to produce unlimited NK cells for cell therapy.[43] One concern regarding immune effector cells derived from iPSCs is how iPSC-derived NK cells compare to their natural counterparts in terms of receptor expression and functional potential. Parallel phenotypic assessment of iPSC-NK and PB-NK revealed highly similar phenotypes, except for higher expression of NKG2A in iPSC-NK cells.[43] NKG2A is typically expressed on more immature NK cells and has been shown to restrict targeting of tumor cells that express high levels of HLA-E.[44] NKG2A is emerging as a major checkpoint in cancer immunotherapy so it may be important to develop strategies to limit its expression on iPSC-derived NK cells. iPSC-NK cells also express less KIR, reflecting the fact that KIR acquisition is a relatively late event during NK cell differentiation and that it is challenging to achieve terminal differentiation of iPSC-NK cells.[45] Whether the lack of KIR expression may be an advantage in terms of less inhibition during the effector phase, or constitute a problem due to lack of KIR-mediated education remains unclear. Likewise, it remains unknown how KIR genetics, including variation in KIR gene content and copy number influence the iPSC-NK repertoire [46, 47]. Although these phenotypic traits suggest that there may be room to further improve iPSC-NK cell differentiation protocols, current evidence suggests that iPSC-NK cells are highly functional in their native state.[43, 48, 49] iPSC-NKs show effective killing of both HLA-deficient and HLA 
sufficient targets including K562, SKOV-3 and SW480. In fact, the efficiency of iPSC-NK cells was found to be greater than that of PB-NK cells in all lines except K562 where the killing efficiency was similar. [50] in vivo studies demonstrate iPSC-NK cells have similar improved activity compared to PB-NK cells against ovarian cell lines MA148 and A1847.[49] Further investigation of the transcriptional regulation of effector function during terminal NK cell differentiation may pave the way for new strategies to promote differentiation and obtain more mature and functional iPSC-NK cells. Likewise, insights into the molecular mechanisms that determine NK cell education may foster new strategies to engineer iPSC-NK cells for increased effector potential.

\section{iPSC-NK cell specificity}

Whereas unmanipulated iPSC-NK cells provide great promise to treatment of diverse tumor types and particularly against HLA loss variants,[22] there is even more exciting potential to use iPSC-NK cells as a template for genetic modifications that will further improve their antitumor activity, such as targeting more resistant tumors using chimeric antigen receptors. (Figure 1) $[48,51,52]$ The success of chimeric antigen receptors (CARs) that redirect T- cell specificity in treatment of hematological cancers has spurred interest in the development of CARs in NK cells. [53-55] CARs are synthetic receptors composed of three parts: ectodomain, transmembrane domain, and endodomain. The ectodomain is composed of the single chain variable fragment $(\mathrm{scFv})$ derived from a monoclonal antibody or an antigen-binding fragment (Fab) which directs antigen recognition specificity either targeting antigens that are overexpressed or antigens that are tumor specific.[56] The scFv can be fused to numerous intracellular signaling domain(s). Connecting the ectodomain to the transmembrane domain is the hinge region. The intracellular domain, or endodomain directs the functionality of the CAR. Since its discovery, CARs have undergone extensive development and there are currently four generations of CARs. In the first generation, the signaling domain is composed of an activation domain which in T cells the $\zeta$ of the T cell receptor complex CD3 was first tested. [52] In second generation CARs, co-stimulatory signaling domain and activation domain act as a dualsignaling domain to increase $\mathrm{T}$ cell activation and proliferation. The addition of a dual-signaling domain has also shown to increase persistence in vivo.[56-58] The signaling domain of third generation CARs have two co-stimulatory domains in addition to the activation domain in order to increase persistence and cytolytic function beyond what was achieved with second generation 
CARs.[59-62] Finally, the fourth generation CARs, or so called armored CARs, carry a payload in the form of a cytokine or even a checkpoint inhibitor, secreted into the tumor environment.[63]

We recently examined the impact of NK subset diversity on CAR function in vitro.[64] We found that the intrinsic potential of the cell, determined both by differentiation and education by self KIR, had an impact on the functional potential of the CAR-engineered NK cells. So called adaptive NK cells, also referred to as memory NK cells, [65] having increased cytotoxic payload and potent effector function,[66, 67] displayed the strongest functional responses following engineering with a third generation CD19-specific CAR. Moreover, CAR engineering did not affect NK receptor repertoire and CAR-NK cells retained their intrinsic capacity to recognize and lyse tumor cells independent of the presence of CAR target antigen. Thus, CAR-NK cells could possibly dampen tumor growth even in the event of antigen loss variants, for example downregulation of CD19 during CAR-T cell therapy.[68]

In another set of recent studies, we designed a novel set of NK cell-specific CARs expressed in iPSC-derived NK cells.[48] Here, we targeted ovarian cancer cells in vivo using an anti-mesothelin (meso) CARs. After testing 10 different CAR constructs with different transmembrane (TM) and signaling domains, including a "conventional" $3^{\text {rd }}$ generation $\mathrm{T}$ cell

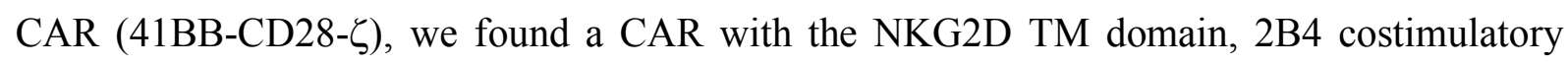

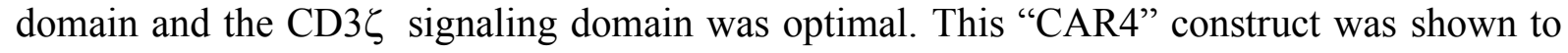
stimulate key NK cell signaling pathways including DAP10 activation, increased Syk activity, phosphorylation of PLC- $\gamma 1$ and PCL- $\gamma 2$, as well as activation of the NFאB pathway. These NK-CAR-iPSC NK cells demonstrated improved killing of human ovarian cancer cells in a murine xenograft model, including prolonged survival and expansion of the CAR-NK cells in vivo. Interestingly, direct comparison of NK-CAR-iPSC NK cells to "conventional" anti-meso CAR-T cells found the CAR-NK cells had less toxicity and improved survival.

In addition to CAR-mediated specificity, CD16 expression can be used as a universal targeting approach when combined with therapeutic monoclonal antibodies such as Herceptin, Erbitux and Rituxan. Through the mAb-mediated targeting, CD16 elicits antibody-directed cellular-cytotoxicity (ADCC) that can effectively eliminate the targeted cells. Because CD16 sheds upon activation, modifications have been made to prevent the stimulation mediated shedding to continue signaling and improve function.[69] Taken together, these findings suggest that one may achieve greater clinical efficacy by combining strategies to enhance the 
intrinsic functional potential of iPSC-NK cells with careful CAR or CD16 designs using optimized signaling motifs that tap into specific NK cell signaling pathways.

\section{iPSC-NK cell stealth and persistence}

One key area of interest for the development of successful off-the-shelf cell therapy is to identify strategies to improve persistence of allogeneic cell therapy products. Importantly, over a decade of studies using various PB-NK cells following conditioning chemotherapy such as cyclophosphamide and fludarabine, plus recipient treatment with interleukin (IL)-2 following NK cell infusion does demonstrate expansion and persistence of the allogeneic NK cells, typically for about 1-3 weeks.[70-72] Heroic efforts are currently being made to create a bank of clinical grade iPSC lines that can be used to treat a variety of disorders in a large number of patients. Donors with homozygous HLA types allow for HLA matching for a majority of recipients.[73] It has been proposed that as few as 50 appropriately selected HLA homozygous donors would provide HLA-matched tissue for over 90\% of the Japanese population. [74] An alternative strategy is to create universal iPSCs by knocking down HLA class I to avoid recognition by $\mathrm{T}$ cells. However, this strategy is unlikely to be successful since HLA class negative cells are rejected by host NK cells. Possible workarounds involve expression of immune suppressive ligands, for example single chains of $\beta 2 \mathrm{~m}$ linked to the non-classical HLAE in complex with carefully selected leader peptides to shut down NKG2A expressing NK cells and yet avoid T cell recognition of HLA-E.[75] However, expression of HLA-E would only protect the cells from the subset of NK cells that actually express NKG2A, which is roughly $50 \%$ of the NK cell population.[76] Moreover, knock down of HLA leads to a rather rapid loss of function in NK cells due to disarming.[77] Alternative strategies are needed perhaps acting at multiple levels to generate universal cells that combine three critical features i) escape from $\mathrm{T}$ cells, ii) escape from NK cells, iii) maintained education.

In addition to avoiding immediate rejection, persistence may also be improved by exogenous or endogenous cytokines. Cytokines play a crucial role in NK cell development, differentiation and homeostasis. Therefore, one of the hurdles to be overcome in NK cell immunotherapy is sustaining in vivo proliferation and persistence of NK cells post infusion. Interleukin 2 (IL-2) has been one the most commonly studied cytokine in a clinical setting, as it was the first growth factor and activation factor described for NK cells. Systemic 
administration of IL-2 is associated with high toxicity but also to the expansion of T regulatory cells (Tregs), which down-regulates NK cell function.[78-80] IL-15 is a pleiotropic cytokine that plays a crucial role during development and maintenance of NK cells.[80, 81] To be fully effective, IL-15 must be trans-presented to IL-15 receptor alpha (IL-15R $\alpha$ ). In order to harness the effects of IL-15 on NK cell expansion and survival, ALT-803, a fusion protein that contains a mutated high-affinity IL-15 linked to IL15R $\alpha$ sushi domain and fused with an Fc domain was created and has entered clinical trials for solid tumors and relapsed hematological cancers.[82, 83] The first results demonstrate increased expansion of NK cells with less toxicity for $19 \%$ of patients that had relapsed after hematopoietic stem cell transplantation.[83] Cytokines are also able to change the chemokine receptor repertoire and boost their migration towards sites of inflammation.[84] Retroviral transduction of NK cells to produce IL-2 and IL-15 has been shown to increase in vivo persistence and activation of NK cells in tumor-bearing mice. [85] Similarly, retroviral transduction of a CD19 CAR and IL15 in ex vivo expanded NK cells showed a significant increase in the in vivo persistence and activation of CAR-NK cells in a mouse leukemia model. [51, 86] Apart from increased in vivo expansion and persistence, such self-supported NK cells may attain a higher level of functionality and at the same time avoid the toxicities associated with high doses of systemic IL-15. An interesting question is whether NK cells that carry an IL-15 transgene are rescued from cell death due to cytokine withdrawal associated with adoptive transfer of the in vitro expanded NK cells.[87]

\section{iPSC-NK cell homing}

Throughout development, acquisition of activating and inhibitory receptors increases NK cell effector capability, while the changes in expression of adhesion molecules and chemokine receptors changes NK cell migration patterns.[27] Activation and expansion of NK cells ex vivo alters the chemokine receptor repertoire of NK cells which may affect the ability of NK cells to migrate to tumor sites. [84] NK cell homing to tumor target sites can also be affected by the changes in expression of chemokines in the tumor microenvironment (TME) as tumor cells change its chemokine composition to promote tumor progression, increase vascularization, and recruit immunosuppressive cells. NK cells are generally absent or found in low numbers in the TME of metastatic disease; one study in solid tumors showed that as little as $0.01 \%-0.04 \%$ of adoptively transferred cells could be detected in the TME.[88, 89] Low infiltration likely reflects the poor capability of NK cells to home effectively to tumor target cells.[90, 91] The 
infiltration of NK cells in certain solid tumor cancers correlates with better prognosis.[91-94] Therefore, the ability of the NK cells to reach the TME could greatly affect the outcome of treatment. Chemokine receptor 3 (CXCR3) promotes NK cell adhesion and migration and has been associated with effective homing of NK cells to tumor sites.[95] The three CXCR3 ligands: CXCL9, CXCL10 and CXCL11 are the most important in acting as a chemoattractant to $\mathrm{CXCR}^{+} \mathrm{NK}$ cells.[95] Mouse studies have shown that local injections of Th1-type chemokines, IFN- $\gamma$ or CXCL10, led to an increase of $\mathrm{CXCR}^{+} \mathrm{NK}$ cells in the TME. $[95,96]$ Moreover, lymphocytes can be recruited to the TME by inducing secretion of Th1-type chemokines in dendritic cells by locally injecting Sendai virus particles or PD1 blockade to induce secretion of CXCR3 ligand in myeloid cells when combined with adoptive cell transfer. $[97,98]$ Local radiation treatment has also been shown in increase CXCR3 ligand secretion in an IFN- $\gamma$ dependent manner. [99] Other mouse studies demonstrate a significant increase in NK cell and $\mathrm{T}$ cell infiltration to the tumor site after treatment with the epigenetic modulator PRC2 that inhibits DNA methylation which has been found to suppress secretion of CXCL9 and CXCL10.[100, 101] For the ultimate design of a synthetic killer, engineering unique and tailored homing properties into iPSC-NK cells may allow targeting of solid tumors that are otherwise poorly infiltrated by cytokine-activated NK cells.

Although we commonly think of NK cells in terms of their killing potential, emerging evidence suggest that other functional properties of NK cells, including their directed secretion of cytokines and chemokines contribute an important bridge to adaptive immunity.[102] NK cells participate in the recruitment and activation of APCs and promote Th1 type responses. $[103,104]$ Thus, there is rational to combine NK cells with checkpoint blockade (CPB) therapy to convert "cold" tumors to "hot" tumors and improve CPB therapy.

\section{iPSC-NK cell resistance}

NK cells that eventually reach the TME are faced with harsh metabolic conditions, including hypoxia, acidic $\mathrm{pH}$ and low glucose levels that all affect NK cell function. [105-107] Within the TME, the immunosuppressive cytokine, transforming growth factor- $\beta$ (TGF- $\beta$ ), has a dramatic negative impact on NK cell function. Tregs in the TME suppress NK cell function by releasing TGF- $\beta$ which act partly through C-type lectin receptor CD69 on NK cells.[108] Immunosuppression by TGF- $\beta$ on NK cells is multifaceted. TGF- $\beta$ affects metabolic activity 
and proliferative capacity as well as changing receptor repertoire of NK cells that leads to a decrease in cytotoxic activity. $[109,110]$ TGF- $\beta$ is induced by hypoxia and in turn promotes the proliferation and accumulation of Tregs within the TME. [111] Tumor-derived cytokines convert macrophages into M2 macrophages which further promote tumor progression creating a positive feedback loop and a continual production of immunosuppressive factors such as IL10 and TGF- $\beta$. [112] The hypoxic environment of the TME also preferentially recruits tumor infiltration by Tregs and M2-polarization and directly impacts the degradation of granzyme B in NK cells via autophagy. [107, 113, 114] This in turn affects the subset of NK cells that are most potent killers, CD56 ${ }^{\mathrm{dim}} \mathrm{CD} 16^{+}$which are targeted for apoptosis.[114] Cancer associated fibroblasts (CAFs) are another source of TGF- $\beta$ in the TME. The high levels of TGF- $\beta$ in the TME drives the differentiation of various resident cells such as fibroblasts, mesenchymal stem cells, endothelial and epithelial cells into CAFs.[115, 116] Although CAFs phenotypically resemble normal myofibroblasts, they express CAF specific markers such as fibroblastassociated protein, fibroblast specific protein-1 and platelet derived growth factor. $[117,118]$ CAFs not only aid in tumor progression by secreting factors that promote vascularization, such as platelet derived growth factor and vascular endothelial growth factor, but they also aid in tumor progression by suppressing the immune response by secreting TFG- $\beta$.

The challenges facing adoptively transferred iPSC-NK cells in the TME can be overcome either by pharmacological interventions or through gene-editing strategies that confer resistance to the suppressive factors. Genetic modification of the NK cell line, NK-92, to express a signaling-dead TGF- $\beta$ receptor that is insensitive to TGF- $\beta$ immunosuppression led to decreased tumor proliferation, increased production of IFN- $\gamma$, and increased survival when mice were challenged with Calu-6 lung cancer cells.[119] Another approach to overcome TGF$\beta$ suppression is to use a TGF- $\beta$ receptor kinase inhibitor, LY2157299, which has been shown in mice to enhance cytokine production and control tumor progression. This effect was greater when coupled with adoptive NK cell transfer when compared to treatment of TGF- $\beta$ receptor kinase inhibitor or adoptive NK cells alone.[120] Currently, drug treatment to overcome oxidative stress such as fluorouracil, cyclophosphamide, and doxorubicin, celecoxib and sunitinib effectively target immunosuppressive cells in the TME but act broadly and therefore can lead to negative side effects.[121] Notably, lessons learned from studying subsets of PBNK, where for example the adaptive NK cells are more resistant to immune suppression due to increased expression of ARID5B[122], can be harnessed in the context of designing iPSC-NK products for increased endurance in the TME. 


\section{Programming effector function - Towards a synthetic killer.}

In order for NK cells to be effective they not only need to travel to and survive in the TME, but must also be able to deliver one or more lytic hits, ultimately killing the intended tumor target. One of the key pathways of NK-cell mediated killing is through degranulation., i.e. the release of dense-core secretory lysosomes that contain perforin and granzymes. NK cells are also able mediate target cell death by means of death receptor pathways TRAIL/TRAIL-R and Fas/FasL and these mechanisms operate sequentially albeit with some overlap.[123] The effector program that regulates NK cell killing evolves under the influence of homeostatic cytokines, and metabolic cues and is further shaped by receptor-ligand interactions during NK cell education. We have recently found that educated NK cells express higher levels of granzyme B stored in unique pre-converged dense-core secretory lysosomes. [124] Whereas the gradual increase in effector potential during differentiation are under tight transcriptional regulation, the unique functional phenotype of educated NK cells appears independent of transcription and represent a way for the cell to integrate receptor input into a physical, compartmentalized molecular memory. The increased loads of granzyme B in educated NK cells set the stage for serial killing.[125] Further insights into the cellular mechanisms that defines the development of a molecular memory in NK cells may pave the way for new means to engineer increased effector function into iPSC-NK cells. For example, it may be possible to identify pathways that boost lysosomal biogenesis and reloading of secretory lysosomes to modulate degranulation in NK cells which could increase the efficiency of NK immunotherapy.

\section{Conclusion}

Engineered allogeneic NK cell therapy holds great promise as the next generation immunotherapy for a wide range of cancer types. However, current protocols based on expansion of PB-NK cells are limited by donor variability in expansion efficiency together with a vast phenotypic heterogeneity of the end products. The use iPSCs as a source for unlimited doses of genetically engineered NK cell products opens up the possibility to create clinical cell banks available to patients on demand. Additionally, repeat dosing with these off-the-shelf NK cells may be more effective, especially for treatment of solid tumors. These products can be geared for detection of HLA class I loss variants, to express CAR or Fc-receptors that recognize tumors expressing a given antigen or coated with therapeutic antibodies. By deepening our 
insights into the fundamental mechanisms that regulate NK cell function, trafficking and tumorhost relationships, we can develop a portfolio of iPSC-NK cell products that have greater function, targeted homing capacity and ability to persist in an allogeneic host and in the tumor microenvironment. 
Figure 1. Engineering of a synthetic killer. The figure illustrates the potential of iPSC-derived

Effector function

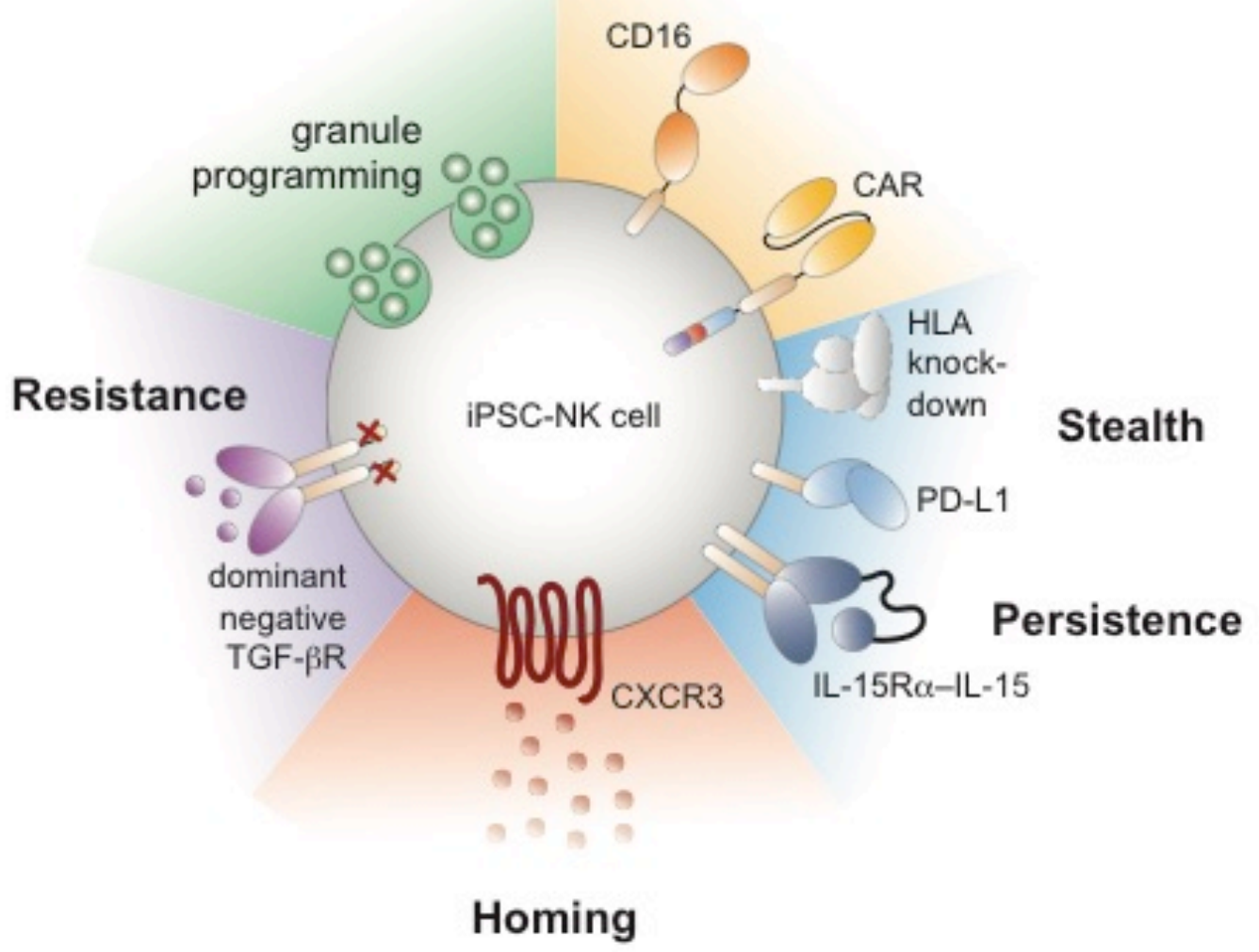

NK cells as a template for engineering. Examples of such gene edits include: i) Strategies aiming to introduce specificity through cleavage-resistant CD16 for combination with therapeutic antibodies or CAR; ii) Strategies aiming at improving persistence of the infused allogeneic iPSC-NK cells by escaping recognition of host $\mathrm{T}$ and NK cells and by making cells self-sufficient in terms of growth factors; iii) Strategies to direct trafficking of iPSC-NK cells to the tumor, here illustrated by overexpression of the chemokine-receptor CXCR3; iv) Strategies to make the cell resistant to immune suppressive factors in the tumor microenvironment, for example by introducing a signaling-dead TGF- $\beta$ receptor; and v) Strategies to improve effector function, for example by modulating the cytotoxic payload. 


\section{References}

1. Nishimura, T., et al., Generation of rejuvenated antigen-specific T cells by reprogramming to pluripotency and redifferentiation. Cell Stem Cell, 2013. 12(1): p. 114-26.

2. Shah, N.N., et al., Acute GVHD in patients receiving IL-15/4-1BBL activated NK cells following T-cell-depleted stem cell transplantation. Blood, 2015. 125(5): p. 784-92.

3. Ando, M. and H. Nakauchi, 'Off-the-shelf' immunotherapy with iPSC-derived rejuvenated cytotoxic Tlymphocytes. Exp Hematol, 2017. 47: p. 2-12.

4. Cooper, L.J., Off-the-shelf T-cell therapy. Blood, 2010. 116(23): p. 4741-3.

5. Themeli, M., I. Riviere, and M. Sadelain, New cell sources for T cell engineering and adoptive immunotherapy. Cell Stem Cell, 2015. 16(4): p. 357-66.

6. Themeli, M., et al., Generation of tumor-targeted human Tlymphocytes from induced pluripotent stem cells for cancer therapy. Nat Biotechnol, 2013. 31(10): p. 928-33.

7. Torikai, H. and L.J. Cooper, Translational Implications for Off-the-shelf Immune Cells Expressing Chimeric Antigen Receptors. Mol Ther, 2016. 24(7): p. 1178-86.

8. Björklund, A., et al., Complete Remission and Signs of Immunoediting Following Haploidentical NK Cell Theray in Refractory High-Risk MDS and AML Ell Therapy in Refractory High-Risk MDS and AML. 2017. 130(Suppl 1): p. 4458.

9. Curti, A., et al., Successful transfer of alloreactive haploidentical KIR ligand-mismatched natural killer cells after infusion in elderly high risk acute myeloid leukemia patients. Blood, 2011. 118(12): p. 3273-9.

10. Ruggeri, L., et al., Effectiveness of donor natural killer cell alloreactivity in mismatched hematopoietic transplants. Science, 2002. 295(5562): p. 2097-100.

11. Wu, J. and L.L. Lanier, Natural killer cells and cancer. Adv Cancer Res, 2003. 90: p. 127-56.

12. Lee, S.H., T. Miyagi, and C.A. Biron, Keeping NK cells in highly regulated antiviral warfare. Trends Immunol, 2007. 28(6): p. 252-9.

13. Kiessling, R., et al., "Natural" killer cells in the mouse. II. Cytotoxic cells with specificity for mouse Moloney leukemia cells. Characteristics of the killer cell. Eur J Immunol, 1975. 5(2): p. 117-21.

14. Kiessling, R., E. Klein, and H. Wigzell, "Natural" killer cells in the mouse. I. Cytotoxic cells with specificity for mouse Moloney leukemia cells. Specificity and distribution according to genotype. Eur J Immunol, 1975. 5(2): p. 112-7.

15. Herberman, R.B., et al., Natural cytotoxic reactivity of mouse lymphoid cells against syngeneic and allogeneic tumors. II. Characterization of effector cells. Int J Cancer, 1975. 16(2): p. 230-9.

16. Herberman, R.B., M.E. Nunn, and D.H. Lavrin, Natural cytotoxic reactivity of mouse lymphoid cells against syngeneic acid allogeneic tumors. I. Distribution of reactivity and specificity. Int J Cancer, 1975. 16(2): p. 216-29.

17. Karre, K., How to recognize a foreign submarine. Immunol Rev, 1997. 155: p. 5-9.

18. Ljunggren, H.G. and K. Karre, In search of the 'missing self': MHC molecules and NK cell recognition. Immunol Today, 1990. 11(7): p. 237-44.

19. Karre, K., NK cells, MHC class I molecules and the missing self. Scand J Immunol, 2002. 55(3): p. 221-8.

20. Karre, K., Natural killer cell recognition of missing self. Nat Immunol, 2008. 9(5): p. 477-80.

21. Elliott, J.M. and W.M. Yokoyama, Unifying concepts of MHC-dependent natural killer cell education. Trends Immunol, 2011. 32(8): p. 364-72.

22. Malmberg, K.J., et al., Immune selection during tumor checkpoint inhibition therapy paves way for NK-cell "missing self" recognition. Immunogenetics, 2017. 69(8-9): p. 547-556.

23. Chaplin, D.D., Overview of the immune response. J Allergy Clin Immunol, 2010. 125(2 Suppl 2): p. S3-23. 
24. Colucci, F., M.A. Caligiuri, and J.P. Di Santo, What does it take to make a natural killer? Nat Rev Immunol, 2003. 3(5): p. 413-25.

25. Smyth, M.J., et al., New aspects of natural-killer-cell surveillance and therapy of cancer. Nat Rev Cancer, 2002. 2(11): p. 850-61.

26. Vivier, E., J.A. Nunes, and F. Vely, Natural killer cell signaling pathways. Science, 2004. 306(5701): p. 1517-9.

27. Goodridge, J.P., B. Önfelt, and K.J. Malmberg, Newtonian cell interactions shape natural killer cell education. Immunol Rev, 2015. 267(1): p. 197-213.

28. Takahashi, K. and S. Yamanaka, Induction of pluripotent stem cells from mouse embryonic and adult fibroblast cultures by defined factors. Cell, 2006. 126(4): p. 663-76.

29. Yu, J., et al., Induced pluripotent stem cell lines derived from human somatic cells. Science, 2007. 318(5858): p. 1917-20.

30. Ghosh, Z., et al., Persistent donor cell gene expression among human induced pluripotent stem cells contributes to differences with human embryonic stem cells. PLoS One, 2010. 5(2): p. e8975.

31. Marchetto, M.C., et al., Transcriptional signature and memory retention of human-induced pluripotent stem cells. PLoS One, 2009. 4(9): p. e7076.

32. Chin, M.H., et al., Induced pluripotent stem cells and embryonic stem cells are distinguished by gene expression signatures. Cell Stem Cell, 2009. 5(1): p. 111-23.

33. Ohi, Y., et al., Incomplete DNA methylation underlies a transcriptional memory of somatic cells in human iPS cells. Nat Cell Biol, 2011. 13(5): p. 541-9.

34. Lister, R., et al., Hotspots of aberrant epigenomic reprogramming in human induced pluripotent stem cells. Nature, 2011. 471(7336): p. 68-73.

35. Deng, J., et al., Targeted bisulfite sequencing reveals changes in DNA methylation associated with nuclear reprogramming. Nat Biotechnol, 2009. 27(4): p. 353-60.

36. Doi, A., et al., Differential methylation of tissue- and cancer-specific CpG island shores distinguishes human induced pluripotent stem cells, embryonic stem cells and fibroblasts. Nat Genet, 2009. 41(12): p. 1350-3.

37. Kim, K., et al., Donor cell type can influence the epigenome and differentiation potential of human induced pluripotent stem cells. Nat Biotechnol, 2011. 29(12): p. 1117-9.

38. Guenther, M.G., et al., Chromatin structure and gene expression programs of human embryonic and induced pluripotent stem cells. Cell Stem Cell, 2010. 7(2): p. 249-57.

39. Newman, A.M. and J.B. Cooper, Lab-specific gene expression signatures in pluripotent stem cells. Cell Stem Cell, 2010. 7(2): p. 258-62.

40. Woll, P.S., et al., Human embryonic stem cell-derived NK cells acquire functional receptors and cytolytic activity. J Immunol, 2005. 175(8): p. 5095-103.

41. Woll, P.S., et al., Human embryonic stem cells differentiate into a homogeneous population of natural killer cells with potent in vivo antitumor activity. Blood, 2009. 113(24): p. 6094-101.

42. Valamehr, B., et al., Platform for induction and maintenance of transgene-free hiPSCs resembling ground state pluripotent stem cells. Stem Cell Reports, 2014. 2(3): p. 366-81.

43. Knorr, D.A., et al., Clinical-scale derivation of natural killer cells from human pluripotent stem cells for cancer therapy. Stem Cells Transl Med, 2013. 2(4): p. 274-83.

44. Nguyen, S., et al., HLA-E upregulation on IFN-gamma-activated AML blasts impairs CD94/NKG2A-dependent NK cytolysis after haplo-mismatched hematopoietic SCT. Bone Marrow Transplant, 2009. 43(9): p. 693-9.

45. Björkström, N.K., et al., Expression patterns of NKG2A, KIR, and CD57 define a process of CD56dim NK-cell differentiation uncoupled from NK-cell education. Blood, 2010. 116(19): $\mathrm{p}$. 3853-64.

46. Beziat, V., et al., Influence of KIR gene copy number on natural killer cell education. Blood, 2013. 121(23): p. 4703-7.

47. Béziat, V., et al., Tracing dynamic expansion of human NK-cell subsets by high-resolution analysis of KIR repertoires and cellular differentiation. Eur J Immunol, 2014. 44(7): p. 2192-6. 
48. Li, Y., et al., Human iPSC-Derived Natural Killer Cells Engineered with Chimeric Antigen Receptors Enhance Anti-tumor Activity. Cell Stem Cell, 2018. 23(2): p. 181-192 e5.

49. Hermanson, D.L., et al., Induced Pluripotent Stem Cell-Derived Natural Killer Cells for Treatment of Ovarian Cancer. Stem Cells, 2016. 34(1): p. 93-101.

50. Zeng, J., et al., Generation of "Off-the-Shelf" Natural Killer Cells from Peripheral Blood CellDerived Induced Pluripotent Stem Cells. Stem Cell Reports, 2017. 9(6): p. 1796-1812.

51. Daher, M. and K. Rezvani, Next generation natural killer cells for cancer immunotherapy: the promise of genetic engineering. Curr Opin Immunol, 2018. 51: p. 146-153.

52. Hermanson, D.L. and D.S. Kaufman, Utilizing chimeric antigen receptors to direct natural killer cell activity. Front Immunol, 2015. 6: p. 195.

53. Sadelain, M., I. Riviere, and R. Brentjens, Targeting tumours with genetically enhanced $T$ lymphocytes. Nat Rev Cancer, 2003. 3(1): p. 35-45.

54. Brentjens, R.J., et al., CD19-targeted T cells rapidly induce molecular remissions in adults with chemotherapy-refractory acute lymphoblastic leukemia. Sci Transl Med, 2013. 5(177): p. 177 ra38.

55. Grupp, S.A., et al., Chimeric antigen receptor-modified T cells for acute lymphoid leukemia. N Engl J Med, 2013. 368(16): p. 1509-1518.

56. Sadelain, M., R. Brentjens, and I. Riviere, The promise and potential pitfalls of chimeric antigen receptors. Curr Opin Immunol, 2009. 21(2): p. 215-23.

57. Maher, J., et al., Human T-lymphocyte cytotoxicity and proliferation directed by a single chimeric TCRzeta /CD28 receptor. Nat Biotechnol, 2002. 20(1): p. 70-5.

58. Brentjens, R.J., et al., Genetically targeted $T$ cells eradicate systemic acute lymphoblastic leukemia xenografts. Clin Cancer Res, 2007. 13(18 Pt 1): p. 5426-35.

59. Till, B.G., et al., CD20-specific adoptive immunotherapy for lymphoma using a chimeric antigen receptor with both CD28 and 4-1BB domains: pilot clinical trial results. Blood, 2012. 119(17): p. 3940-50.

60. Pule, M.A., et al., A chimeric T cell antigen receptor that augments cytokine release and supports clonal expansion of primary human T cells. Mol Ther, 2005. 12(5): p. 933-41.

61. Carpenito, C., et al., Control of large, established tumor xenografts with genetically retargeted human T cells containing CD28 and CD137 domains. Proc Natl Acad Sci U S A, 2009. 106(9): p. 3360-5.

62. Zhong, X.S., et al., Chimeric antigen receptors combining 4-1BB and CD28 signaling domains augment PI3kinase/AKT/BCl-XL activation and CD8+T cell-mediated tumor eradication. Mol Ther, 2010. 18(2): p. 413-20.

63. Yeku, O.O. and R.J. Brentjens, Armored CAR T-cells: utilizing cytokines and pro-inflammatory ligands to enhance CAR T-cell anti-tumour efficacy. Biochem Soc Trans, 2016. 44(2): p. 412-8.

64. Oei, V.Y.S., et al., Intrinsic Functional Potential of NK-Cell Subsets Constrains Retargeting Driven by Chimeric Antigen Receptors. Cancer Immunol Res, 2018. 6(4): p. 467-480.

65. Paust, S., C.A. Blish, and R.K. Reeves, Redefining Memory: Building the Case for Adaptive NK Cells. J Virol, 2017. 91(20).

66. Liu, L.L., et al., Ex Vivo Expanded Adaptive NK Cells Effectively Kill Primary Acute Lymphoblastic Leukemia Cells. Cancer Immunol Res, 2017. 5(8): p. 654-665.

67. Liu, L.L., et al., Harnessing adaptive natural killer cells in cancer immunotherapy. Mol Oncol, 2015. 9(10): p. 1904-17.

68. Gardner, R., et al., Acquisition of a CD19-negative myeloid phenotype allows immune escape of MLL-rearranged B-ALL from CD19 CAR-T-cell therapy. Blood, 2016. 127(20): p. 2406-10.

69. Jing, Y., et al., Identification of an ADAM17 cleavage region in human CD16 (FcgammaRIII) and the engineering of a non-cleavable version of the receptor in NK cells. PLoS One, 2015. 10(3): p. e0121788.

70. Romee, R., et al., Cytokine-induced memory-like natural killer cells exhibit enhanced responses against myeloid leukemia. Sci Transl Med, 2016. 8(357): p. 357ra123. 
71. Bachanova, V., et al., Clearance of acute myeloid leukemia by haploidentical natural killer cells is improved using IL-2 diphtheria toxin fusion protein. Blood, 2014. 123(25): p. 3855-63.

72. Miller, J.S., et al., Successful adoptive transfer and in vivo expansion of human haploidentical NK cells in patients with cancer. Blood, 2005. 105(8): p. 3051-7.

73. Taylor, C.J., et al., Banking on human embryonic stem cells: estimating the number of donor cell lines needed for HLA matching. Lancet, 2005. 366(9502): p. 2019-25.

74. Nakatsuji, N., F. Nakajima, and K. Tokunaga, HLA-haplotype banking and iPS cells. Nat Biotechnol, 2008. 26(7): p. 739-40.

75. Gornalusse, G.G., et al., HLA-E-expressing pluripotent stem cells escape allogeneic responses and lysis by NK cells. Nat Biotechnol, 2017.

76. Fauriat, C., et al., Estimation of the size of the alloreactive NK cell repertoire: studies in individuals homozygous for the group A KIR haplotype. J Immunol, 2008. 181(9): p. 6010-9.

77. Boudreau, J.E., et al., Cell-Extrinsic MHC Class I Molecule Engagement Augments Human NK Cell Education Programmed by Cell-Intrinsic MHC Class I. Immunity, 2016. 45(2): p. 280-91.

78. Rosenberg, S.A., et al., A progress report on the treatment of 157 patients with advanced cancer using lymphokine-activated killer cells and interleukin-2 or high-dose interleukin-2 alone. N Engl J Med, 1987. 316(15): p. 889-97.

79. Ghiringhelli, F., et al., $C D 4+C D 25+$ regulatory $T$ cells inhibit natural killer cell functions in a transforming growth factor-beta-dependent manner. J Exp Med, 2005. 202(8): p. 1075-85.

80. Waldmann, T.A., S. Dubois, and Y. Tagaya, Contrasting roles of IL-2 and IL-15 in the life and death of lymphocytes: implications for immunotherapy. Immunity, 2001. 14(2): p. 105-10.

81. Fehniger, T.A. and M.A. Caligiuri, Interleukin 15: biology and relevance to human disease. Blood, 2001. 97(1): p. 14-32.

82. Wrangle, J.M., et al., ALT-803, an IL-15 superagonist, in combination with nivolumab in patients with metastatic non-small cell lung cancer: a non-randomised, open-label, phase $1 \mathrm{~b}$ trial. Lancet Oncol, 2018. 19(5): p. 694-704.

83. Romee, R., et al., First-in-human Phase 1 Clinical Study of the IL-15 Superagonist Complex ALT-803 to Treat Relapse after Transplantation. Blood, 2018.

84. Beider, K., et al., Involvement of CXCR4 and IL-2 in the homing and retention of human NK and NK T cells to the bone marrow and spleen of NOD/SCID mice. Blood, 2003. 102(6): p. 1951-8.

85. Groth, A., et al., Mechanisms of tumor and viral immune escape from natural killer cellmediated surveillance. J Innate Immun, 2011. 3(4): p. 344-54.

86. Liu, E., et al., Cord blood NK cells engineered to express IL-15 and a CD19-targeted CAR show long-term persistence and potent antitumor activity. Leukemia, 2018. 32(2): p. 520-531.

87. Mao, Y., et al., IL-15 activates mTOR and primes stress-activated gene expression leading to prolonged antitumor capacity of NK cells. Blood, 2016. 128(11): p. 1475-89.

88. Fisher, B., et al., Tumor localization of adoptively transferred indium-111 labeled tumor infiltrating lymphocytes in patients with metastatic melanoma. J Clin Oncol, 1989. 7(2): p. 250-61.

89. Pockaj, B.A., et al., Localization of 111indium-labeled tumor infiltrating lymphocytes to tumor in patients receiving adoptive immunotherapy. Augmentation with cyclophosphamide and correlation with response. Cancer, 1994. 73(6): p. 1731-7.

90. Vujanovic, N.L., et al., Antitumor Functions of Natural Killer Cells and Control of Metastases. Methods, 1996. 9(2): p. 394-408.

91. Villegas, F.R., et al., Prognostic significance of tumor infiltrating natural killer cells subset CD57 in patients with squamous cell lung cancer. Lung Cancer, 2002. 35(1): p. 23-8.

92. Coca, S., et al., The prognostic significance of intratumoral natural killer cells in patients with colorectal carcinoma. Cancer, 1997. 79(12): p. 2320-8.

93. Ishigami, S., et al., Prognostic value of intratumoral natural killer cells in gastric carcinoma. Cancer, 2000. 88(3): p. 577-83. 
94. Rusakiewicz, S., et al., Immune infiltrates are prognostic factors in localized gastrointestinal stromal tumors. Cancer Res, 2013. 73(12): p. 3499-510.

95. Wennerberg, E., et al., CXCL10-induced migration of adoptively transferred human natural killer cells toward solid tumors causes regression of tumor growth in vivo. Cancer Immunol Immunother, 2015. 64(2): p. 225-35.

96. Wendel, M., et al., Natural killer cell accumulation in tumors is dependent on IFN-gamma and CXCR3 ligands. Cancer Res, 2008. 68(20): p. 8437-45.

97. Peng, W., et al., PD-1 blockade enhances T-cell migration to tumors by elevating IFN-gamma inducible chemokines. Cancer Res, 2012. 72(20): p. 5209-18.

98. Fujihara, A., et al., Intratumoral injection of inactivated Sendai virus particles elicits strong antitumor activity by enhancing local CXCL10 expression and systemic NK cell activation. Cancer Immunol Immunother, 2008. 57(1): p. 73-84.

99. Lugade, A.A., et al., Radiation-induced IFN-gamma production within the tumor microenvironment influences antitumor immunity. J Immunol, 2008. 180(5): p. 3132-9.

100. Peng, D., et al., Epigenetic silencing of TH1-type chemokines shapes tumour immunity and immunotherapy. Nature, 2015. 527(7577): p. 249-53.

101. Nagarsheth, N., et al., PRC2 Epigenetically Silences Th1-Type Chemokines to Suppress Effector T-Cell Trafficking in Colon Cancer. Cancer Res, 2016. 76(2): p. 275-82.

102. Bottcher, J.P., et al., NK Cells Stimulate Recruitment of CDC1 into the Tumor Microenvironment Promoting Cancer Immune Control. Cell, 2018. 172(5): p. 1022-1037 e14.

103. Gill, R.G., NK cells: elusive participants in transplantation immunity and tolerance. Curr Opin Immunol, 2010. 22(5): p. 649-54.

104. Pallmer, K. and A. Oxenius, Recognition and Regulation of T Cells by NK Cells. Front Immunol, 2016. 7: p. 251.

105. Lardner, A., The effects of extracellular pH on immune function. J Leukoc Biol, 2001. 69(4): p. 522-30.

106. Muller, B., B. Fischer, and W. Kreutz, An acidic microenvironment impairs the generation of non-major histocompatibility complex-restricted killer cells. Immunology, 2000. 99(3): p. 37584.

107. Noman, M.Z., et al., Microenvironmental hypoxia orchestrating the cell stroma cross talk, tumor progression and antitumor response. Crit Rev Immunol, 2011. 31(5): p. 357-77.

108. Terme, M., et al., Regulatory T cells control dendritic cell/NK cell cross-talk in lymph nodes at the steady state by inhibiting CD4+ self-reactive T cells. J Immunol, 2008. 180(7): p. 4679-86.

109. Li, M.O., et al., Transforming growth factor-beta regulation of immune responses. Annu Rev Immunol, 2006. 24: p. 99-146.

110. Viel, S., et al., TGF-beta inhibits the activation and functions of $N K$ cells by repressing the mTOR pathway. Sci Signal, 2016. 9(415): p. ra19.

111. Deng, B., et al., Intratumor hypoxia promotes immune tolerance by inducing regulatory $T$ cells via TGF-beta1 in gastric cancer. PLoS One, 2013. 8(5): p. e63777.

112. Quatromoni, J.G. and E. Eruslanov, Tumor-associated macrophages: function, phenotype, and link to prognosis in human lung cancer. Am J Transl Res, 2012. 4(4): p. 376-89.

113. Baginska, J., et al., Granzyme B degradation by autophagy decreases tumor cell susceptibility to natural killer-mediated lysis under hypoxia. Proc Natl Acad Sci U S A, 2013. 110(43): p. 17450-5.

114. Bauernhofer, T., et al., Preferential apoptosis of CD56dim natural killer cell subset in patients with cancer. Eur J Immunol, 2003. 33(1): p. 119-24.

115. Madar, S., I. Goldstein, and V. Rotter, 'Cancer associated fibroblasts'--more than meets the eye. Trends Mol Med, 2013. 19(8): p. 447-53.

116. Augsten, M., Cancer-associated fibroblasts as another polarized cell type of the tumor microenvironment. Front Oncol, 2014. 4: p. 62.

117. Franco, O.E., et al., Cancer associated fibroblasts in cancer pathogenesis. Semin Cell Dev Biol, 2010. 21(1): p. 33-9. 
118. Rasanen, K. and A. Vaheri, Activation of fibroblasts in cancer stroma. Exp Cell Res, 2010. 316(17): p. 2713-22.

119. Yang, B., et al., Blocking transforming growth factor-beta signaling pathway augments antitumor effect of adoptive NK-92 cell therapy. Int Immunopharmacol, 2013. 17(2): p. 198204.

120. Otegbeye, F., et al., Inhibiting TGF-beta signaling preserves the function of highly activated, in vitro expanded natural killer cells in AML and colon cancer models. PLoS One, 2018. 13(1): p. e0191358.

121. Murray, S. and A. Lundqvist, Targeting the tumor microenvironment to improve natural killer cell-based immunotherapies: On being in the right place at the right time, with resilience. Hum Vaccin Immunother, 2016. 12(3): p. 607-11.

122. Cichocki, F., et al., ARID5B regulates metabolic programming in human adaptive NK cells. J Exp Med, 2018. 215(9): p. 2379-2395.

123. Balzarolo, M., et al., NAB2 and EGR-1 exert opposite roles in regulating TRAIL expression in human Natural Killer cells. Immunol Lett, 2013. 151(1-2): p. 61-7.

124. Goodridge, J.P., et al., Modulation of Secretory Lysosomes During NK Cell Education Leads to Accumulation of Granzyme B and Enhanced Functional Potential. bioRxiv, 2018.

125. Forslund, E., et al., Microchip-Based Single-Cell Imaging Reveals That CD56dimCD57-KIRNKG2A+ NK Cells Have More Dynamic Migration Associated with Increased Target Cell Conjugation and Probability of Killing Compared to CD56dimCD57-KIR-NKG2A-NK Cells. J Immunol, 2015. 195(7): p. 3374-81. 